\title{
Construction and Control Aerial Manipulator Robot
}

\author{
Steeven J. Loor ${ }^{(凶)}$, Alan R. Bejarano ${ }^{(凶)}$, Franklin M. Silva ${ }^{(凶)}$, \\ and Víctor H. Andaluz ${ }^{(凶)}$ \\ Universidad de Las Fuerzas Armadas ESPE, Sangolquí, Ecuador \\ \{sjloor, arbejarano2, fmsilva, vhandaluz1\}@espe.edu.ec
}

\begin{abstract}
This article presents the construction of an aerial manipulator robot composed of one or two robotic arms on an unmanned aerial vehicle, in order to execute control tasks in an autonomous or tele-operated manner. This aerial manipulator robot can work with one or two arms depending on the application requirements. The arms have been designed to serve several purposes: object manipulation and protect the actuating servos against direct impacts and overloads. Finally, a trajectory tracking algorithm is implemented and the simulation results are presented and discussed, which validate the controller and the proposed modeling.
\end{abstract}

Keywords: UAV $\cdot$ Robotic arms $\cdot$ Aerial manipulator robot

\section{Introduction}

During the last decades, research in robotics has been oriented to find solutions to the technical needs of applied robotics. Today, the creation of new needs and markets outside the traditional manufacturing robotics market (e.g., cleaning, mine clearance, construction, shipbuilding, agriculture) and the world in which we live is demanding field and service robots to serve the new market and human social needs [1]. The International Federation of Robotics defines a service robot as a semi- or fully autonomous operated robot that performs useful services for humans and is not used for manufacturing [2]. They are those robotic systems that help people in their daily lives at work, at home, in leisure, and as part of the assistance to the disabled and the elderly. In other words, any robot used in the medical, health, military, domestic and educational industries is considered a service robot [3]. The UAVs have been widely used in military applications, Applications in agriculture and industrial environments are currently being exploited $[5,6,7,8]$. There are several control strategies for UAVs some of which are energy optimization $[9,10]$ and fuzzy control.

The UAV industry is growing very rapidly due to the use of UAVs in commercial areas. However, unmanned aerial vehicles often do not meet the needs of complicated missions such as object handling, as they are only able to navigate in spaces that are difficult to access. For this reason, it is proposed to incorporate one or two robotic arms on an aerial platform (UAV). The combination of aerial mobile platforms with 
robotic arms is known as aerial mobile manipulators [10]. Airborne manipulators have recently encompassed an extensive area of research, which has focused the attention of multiple research groups and international companies interested in such technology [11]. The objective of these projects is the development of robotic systems with handling capabilities. The most commonly used platforms for air manipulators are the helicopter or multirotor type, in its different variants: quadrotor, hexarotor or octrotor, combined with robotic arms with multiple degrees of freedom [12]. Given the versatility of this type of robotic system, different tasks can be performed, such as: welding [19]; transport of long objects [13]; handling of items placed at a height that is difficult to access [13]; construction [14]; transport of goods [15]; power-line applications [16]; hazardous tasks involving human beings, among others [19, 20, 21].

This application seeks to break the paradigm with respect to the manipulation of objects with the design and construction of robotic arms with $3^{\circ}$ of freedom each one since at the moment there are manipulators with very simple arms with a single degree of freedom, in addition an algorithm of control in closed loop will be implemented to follow a task of control in trajectory and thus to be fortifying and improving the process of autonomous tasks in the different scopes leisure, work, military, among others for example cleaning, demining, construction. Construction and control are the main objectives of this work.

\section{Aerial Manipulator Robot Construction}

The airborne manipulator robot is a system conceived and designed to maintain its stability at different flight heights and under different weather conditions. Therefore, in order to comply with all the functional and safety requirements demanded for this type of equipment, a preliminary design of the drone and the robotic arm Fig. 1 was started and these were modified and optimized in geometry and dimensions during the design process, according to the maximum stresses generated in each component under critical operating conditions, until an adequate safety factor was reached and the definitive design of the air handling robot was obtained.

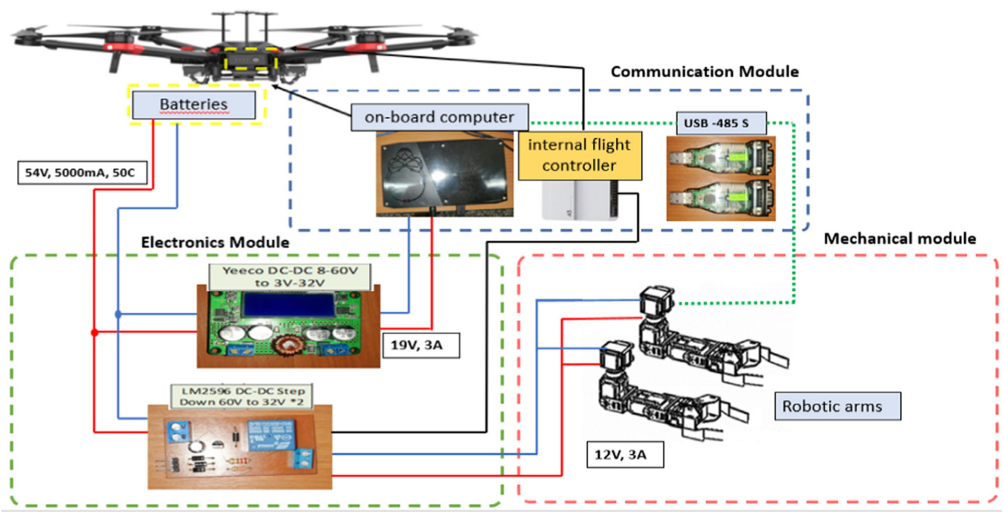

Fig. 1. Electronic, mechanical and communication connection diagram. 


\subsection{Electronics Module}

For the implementation of the air handler, two voltage regulators are included that are supplied by the UAV batteries; one of $54 \mathrm{~V} 7 \mathrm{~A}$ to $18 \mathrm{~V} 3 \mathrm{~A}$ for the computer, and another of $54 \mathrm{~V} 6 \mathrm{~A}$ to $12 \mathrm{~V}$ 6A. The addition of these electronic elements ensures the proper operation of both the computer and the robotic arm and prevents overvoltage and overcurrent in them, for which experimental tests are conducted on power consumption and battery life.

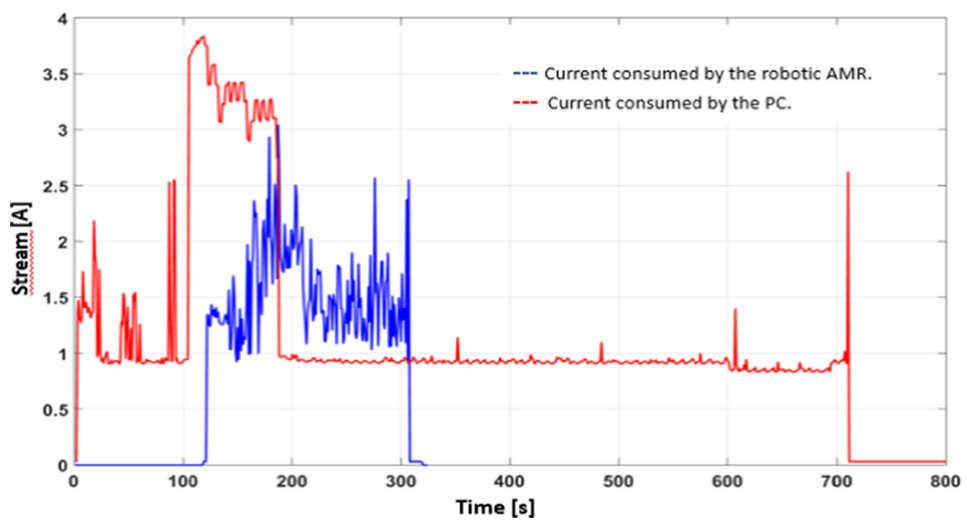

Fig. 2. Power tests of both regulators.

Additionally, an emergency circuit used to suspend erroneous arm actions is included, allowing power to be removed when it could become a hazard to the propellers of the Matrice 600pro, and achieving a safe scheduled landing, this in order to preserve the components and the UAV from an unexpected collision [17].

Finally, several power tests have been carried out to validate the correct operation of the implemented equipment. Figure 2 shows scenarios where both the computer and the robot arm have been subjected to maximum current tests i.e. the computer using all processor cores and the arm with a load of $2 \mathrm{kgs}$.

\subsection{Mechanical Module}

The main structure and the arms of the airborne manipulator robot must be analyzed to verify that they are capable of supporting the weight of the robotic arms and other components that make up the robot: a prototype is made (modeling) with its geometry and weight that simulates the airborne manipulator robot. Afterwards, the structural analysis is carried out by means of finite elements (FEM), with the use of CAD software. Finally, all the necessary mechanical components that make up the air handling robot are integrated. It should be noted that the mechanical design of the base that will serve as a support for the robotic arms is of great importance, and will be assembled on the UAV. This will have the capacity to place one or two robotic arms or just one so that the plate will have 3 couplings distributed evenly so that it does not affect the center of gravity of the UAV. 


\subsection{Bilateral Communication Module}

The air handling system has a hardware remote control, the pilot has the option to intervene or not in basic maneuvers such as take-off and landing. A higher priority was given to pilot commands over autonomous control; in case of unforeseen situations, the pilot can avoid autonomous control and immediately operate the hardware controller.

This approach was implemented in a three-layer architecture in Robotic Operating System (ROS), this simplified its development and look to the future will make possible two things: the creation of a swarm of UAVs and their migration on board the UAV. Increasing the independence of the UAV from the ground control station (GCS).

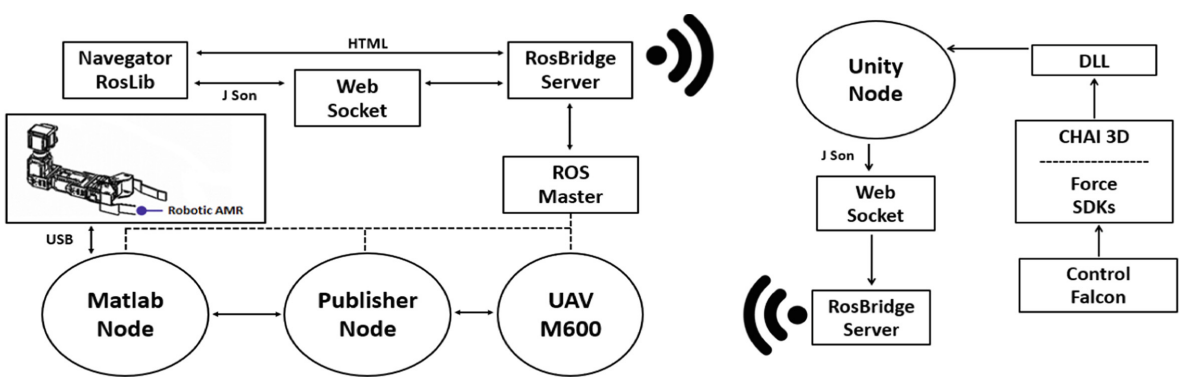

Fig. 3. Communication structure

The structure of this communication system in Fig. 3 uses the Onboard SDK, which has ROS compatibility and this is defined as a node, inside the UAV Matlab computer it runs as a node and with the help of a proprietary node of the Matlab libraries it will send the calculated data and then print the velocities. In the same way through a USB-485 serial communication the arm or arms are controlled depending on the type of configuration being used.

Besides, a ROS Bridge Server is initialized with the purpose that from the Master computer through a FALCOM Force SDK and with the CHAI 3D libraries, it can be read from the libraries to a Unity Node through shared memories (DLL), with this data in the Unity Node it will be written through the Web Socket that will be anchored to the ROS Bridge Server, and it will be possible to send the data from the FALCOM to Matlab that is running in the UAV computer.

\section{Control Algorithm}

The following is a closed-loop autonomous control scheme that validates the construction and the communication channel, which defines a desired task that through an addition node enters the non-linear controller, which sends the control actions to the AMR that is internally distributed in kinematic and dynamic;

PID feedback is performed in each of the segments to pass through the controller and enter a direct kinematics node which closes the loop and compensates errors by entering directly to the non-linear controller [18]. 
To carry out the experimental tests, it is necessary to implement a control law based on the kinematics of the aerial manipulator root described in the previous section, two types of control can be implemented by trajectory and position, in which the trajectory is defined as:

$$
\mathbf{v}_{r e f}(t)=\mathbf{J}^{\#}\left(\dot{\mathbf{h}}_{\mathbf{d}}+\mathbf{K}_{\mathbf{1}} \tanh \left(\mathbf{K}_{2} \tilde{\mathbf{h}}\right)\right)
$$

where $\dot{h}_{d}$ is the reference velocity input of the aerial mobile manipulator for the controller; $\mathbf{J}^{\#}$ is the matrix of pseudoinverse kinematics for the aerial mobile manipulator; while that $\mathbf{K}_{1}>0$ and $\mathbf{K}_{2}>0$ area gain matrix of the controller that weigh the control error respect to the inertial frame $<R>$; and the $\tanh ($.) represents the function saturation of maniobrability velocities in the aerial mobile manipulator [18].

In addition, a position control can only be implemented with: $\dot{\mathbf{h}}_{\mathbf{d}}=0$ so the equation for position control would look like this:

$$
\mathbf{v}_{r e f}(t)=\mathbf{J}^{\#}\left(\mathbf{K}_{\mathbf{1}} \tanh \left(\mathbf{K}_{2} \tilde{\mathbf{h}}\right)\right)
$$

\section{Experimental Results}

This section presents the built AMR, which is composed of a Hexarrotor Matrice-600 and the 3 DOF robot arm, in addition the communication described the results and the proposed controller. In Fig. 4 it is possible to observe the AMR manipulating an object, in this case a pin pon ball, the manipulation was made in experimental tests in laboratory and also in field tests, with this manipulation and flight tests the process of construction and implementation of the arm in the aerial platform is validated. Matlab node that is running on the UAV computer, a sampling period of 100 [ms] will be used).

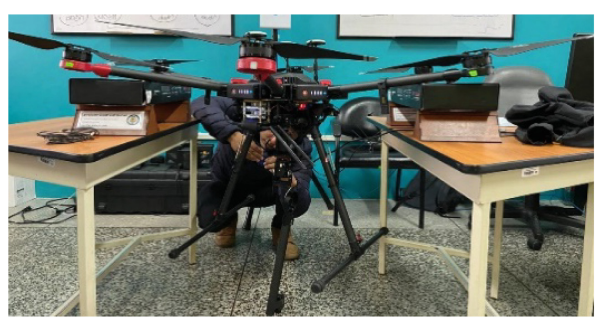

a) Air handler implementation.

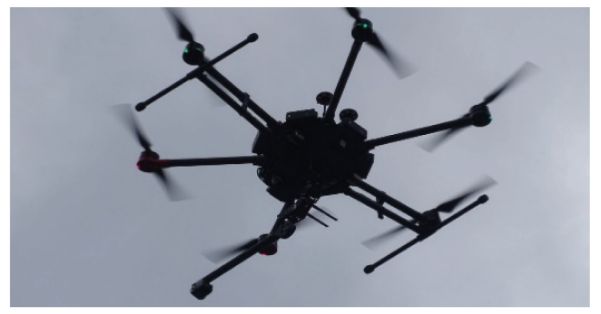

b) Airborne robot with base deployment for greater arm work area

Fig. 4. AMR in operation and stand by.

Figure 5a, shows the desired trajectory and the current trajectory of the end-effector. It can be seen that the proposed controller presents a good performance. Figure $5 \mathrm{c}$, shows the evolution of the tracking errors, which remain close to zero, while Fig. 5b and Fig. 5d show the control actions. 


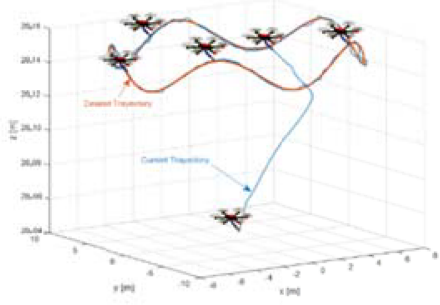

a) Stroboscopic movement in the trajectory tracking.
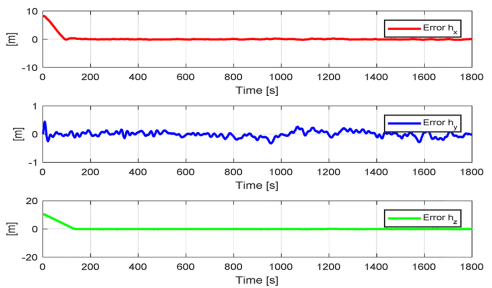

c) Control errors of the end-effector AMR
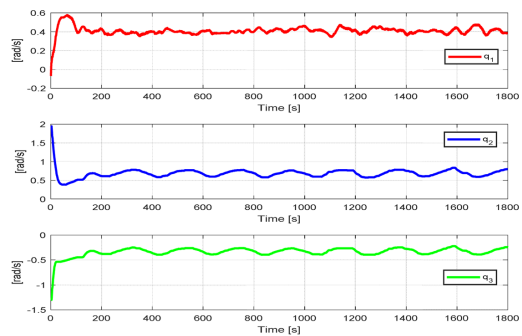

b) Commands of maneuverability of Robotic Arm.
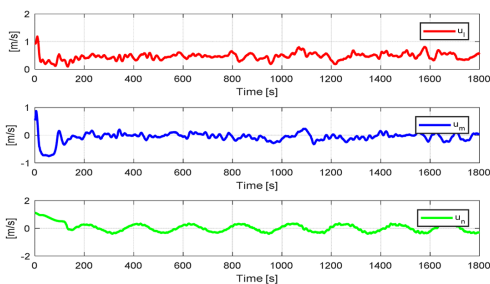

d) Commands of maneuverability of the UAV

Fig. 5. Graphs obtained from the implemented system.

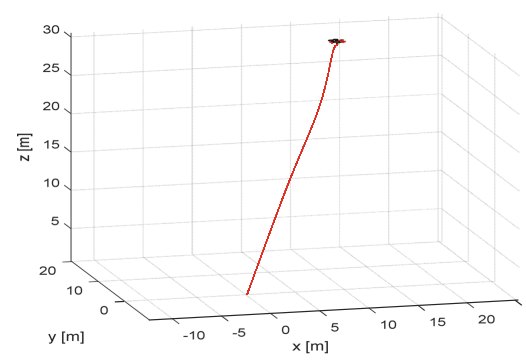

a) Stroboscopic movement with position control.
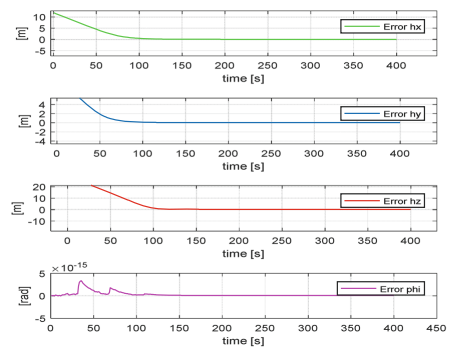

c) Control errors of the end-effector of the aerial manipulator robot
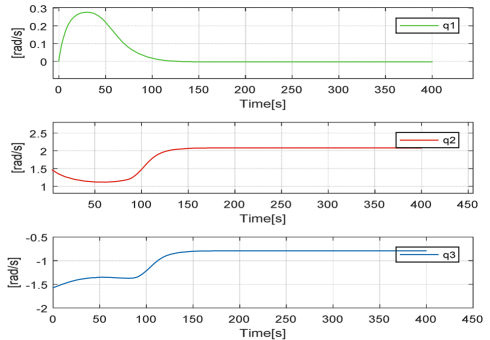

b) Commands of maneuverability of Robotic Arm.
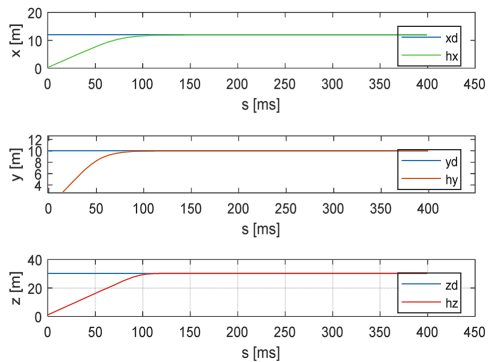

d) Desired and actual positions of the operating end of the air handler

Fig. 6. Graphs obtained from the implemented system with position control. 
Figure 6a, shows the desired position and the current trajectory of the end-effector. Figure $6 \mathrm{c}$, shows the evolution of the tracking errors, which remain close to zero, while Fig. 6b show the control actions and Fig. 6d shows how the UAV reaches the desired positions.

\section{Conclusion}

This document presents a novel aerial manipulator robot that consists of a combination of mechanical and control development strategies, with the ability to have modular arms (one or two arms depending on the application that requires it) and a kinematic controller responsible for carrying out the trajectory tracking task. The main advantage of the control law proposed here lies in its simplicity and ease of application, compared to others already available in the literature. The results of the experimentation have demonstrated the ability of the controller to globally and asymptotically zero the controlled state variables and simultaneously avoid any saturation in the flight commands. However, in order to take full advantage of the proposed approach, further work is needed on motion control and trajectory planning of the position of the end effector.

Acknowledgements. The authors would like to thank the Corporación Ecuatoriana para el Desarrollo de la Investigación and Academia CEDIA for the financing given to research, development, and innovation, through the CEPRA projects, especially the project CEPRA-XIII-2019-08; Sistema colaborativo de robots Aéreos para Manipular Cargas con Optimo Consumo de Recursos; also to Universidad de las Fuerzas Armadas ESPE, Escuela Superior Politécnica de Chimborazo, Universidad Nacional de Chimborazo, Universidad tecnológica Indoamérica, Universidad internacional del Ecuador, Universidad central de Venezuela, and Grupo de Investigación ARSI, for the support to develop this work.

\section{References}

1. Garcia, E., Jimenez, M.A., De Santos, P.G., Armada, M.: The evolution of robotics research. IEEE Robot. Autom. Mag. 14, 1 (2007)

2. International Federation of Robotics. Service robots: Provisional definition of service robots. http://www.ifr.org/service-robots/

3. Moradi, H., et al.: Service robotics (the rise and bloom of service robots) [tc spotlight]. IEEE Robot. Autom. Mag. 20(3), 22-24 (2013)

4. Lee, K., Lee, J., Woo, B., Lee, J., Lee, Y., Ra, S.: Modeling and control of a articulated robot arm with embedded joint actuators. In: 2018 International Conference on Information and Communication Technology Robotics (ICT-ROBOT), Busan, pp. 1-4, (2018)

5. Thai, H.N., Phan, A.T., Nguyen, C.K., Ngo, Q.U., Dinh, P.T., Vo, Q.T.: Trajectory tracking control design for dual-arm robots using dynamic surface controller. In: 2019 First International Symposium on Instrumentation, Control, Artificial Intelligence, and Robotics (ICA-SYMP) (2019). https://doi.org/10.1109/ica-symp.2019.8646243

6. Ruan, L., et al.: Energy-efficient multi-UAV coverage deployment in UAV networks: a gametheoretic framework. China Commun. 15(10), 194-209 (2018)

7. Pajares, G.: Overview and current status of remote sensing applications based on unmanned aerial vehicles (UAVs). Photogramm. Eng. Remote Sens. 81(4), 281-330 (2015) 
8. Ramon Soria, P., Arrue, B., Ollero, A.: Detection, location and grasping objects using a stereo sensor on UAV in outdoor environments. Sensors 17(1), 103 (2017)

9. Suarez, A., Heredia, G., Ollero, A.: Lightweight compliant arm with compliant finger for aerial manipulation and grasping. In: 2016 IEEE/RSJ International Conference on Intelligent Robots and Systems (IROS) (2016)

10. Bartelds, T., Capra, A., Hamaza, S., Stramigioli, S., Fumagalli, M.: Compliant aerial manipulators: Toward a new generation of aerial robotic workers. IEEE Robot. Autom. Lett. 1(1), 477-483 (2016)

11. Tognon, M., Franchi, A.: Dynamics, control, and estimation for aerial robots tethered by cables or bars. IEEE Trans. Rob. 33(4), 834-845 (2017)

12. Suarez, A., Heredia, G., Ollero, A.: Lightweight compliant arm with compliant finger for aerial manipulation and grasping. In 2016 IEEE/RSJ International Conference on Intelligent Robots and Systems (IROS)

13. Suárez, A., Sanchez-Cuevas, P., Fernandez, M., Perez, M., Heredia, G., Ollero, A.: Lightweight and compliant long reach aerial manipulator for inspection operations. In: 2018 IEEE/RSJ International Conference on Intelligent Robots and Systems (IROS), pp. 6746-6752. IEEE, October 2018

14. Marquez, F., Maza, I., Ollero, A.: Comparacion de planificadores de caminos basados en muestro para un robot aereo equipado con brazo manipulador. Comité Español de Automática de la CEA-IFAC (2015)

15. Cano, R., Pérez, C., Pruaño, F., Ollero, A., Heredia, G.: Diseño Mecánico de un Manipulador Aéreo Ligero de 6 GDL para la Construcción de Estructuras de Barras. ARCAS (ICT2011-287617) del séptimo Programa Marco de la Comisión Europea y el proyecto CLEAR (DPI2011-28937-C02-01) (2013)

16. Kim, S., Choi, S., Kim, H.J.: Aerial manipulation using a quadrotor with a two DOF robotic arm. In: IEEE/RSJ International Conference on Intelligent Robots and Systems, Tokyo, pp. 4990-4995 (2013)

17. https://www.dji.com/matrice600-pro

18. Ortiz, J., Erazo, A., Carvajal, C., Pérez, J., Proaño, L., Silva M,F., Andaluz, V.: Modeling and kinematic nonlinear control of aerial mobile manipulators. In: Computational Kinematics, pp. 87-95 (2017)

19. Leica, P., Balseca, J., Cabascango, D., Chávez, D., Andaluz, G., Andaluz, V.H.: Controller based on null space and sliding mode (NSB-SMC) for bidirectional teleoperation of mobile robots formation in an environment with obstacles. In: 2019 IEEE Fourth Ecuador Technical Chapters Meeting (ETCM), Guayaquil, pp. 16 (2019)

20. Varela-Aldás, J., Andaluz, V.H., Chicaiza, F.A.: Modelling and control of a mobile manipulator for trajectory tracking. In: 2018 International Conference on Information Systems and Computer Science (INCISCOS), Quito (2018)

21. Andaluz, V., Rampinelli, V.T.L., Roberti, F., Carelli, R.: Coordinated cooperative control of mobile manipulators. In: 2011 IEEE International Conference on Industrial Technology, Auburn, AL, pp. 300-305 (2011) 